\title{
Assessment of GPS + Galileo and multi-frequency Galileo single-epoch precise positioning with network corrections
}

\author{
Jacek Paziewski • Pawel Wielgosz
}

Received: 20 May 2013/ Accepted: 22 November 2013/Published online: 12 December 2013

(C) The Author(s) 2013. This article is published with open access at Springerlink.com

\begin{abstract}
Several processing strategies that use dual-frequency GPS-only solution, multi-frequency Galileo-only solution, and finally tightly combined dual-frequency GPS + Galileo solution were tested and analyzed for their applicability to single-epoch long-range precise positioning. In particular, a multi-system GPS + Galileo solution was compared to GPS double-frequency solution as well as to Galileo double-, triple-, and quadruple-frequency solutions. Also, the performance of the strategies was analyzed under clear-sky and obstructed satellite visibility in both single-baseline and multi-baseline modes. The results indicate that tightly combined GPS + Galileo instantaneous positioning has a clear advantage over single-system solutions and provides an accurate and reliable solution. It was also confirmed that application of multi-frequency observations in case of Galileo system has an advantage over a dual-frequency solution.
\end{abstract}

Keywords Precise satellite positioning .

Multi-frequency observations · GPS · Galileo · GNSS

\section{Introduction}

The key factor in relative positioning is the resolution of double-differenced ambiguities. Generally, for short observing sessions, a reliable ambiguity resolution is more difficult. However, the most challenging task is the correct ambiguity resolution using data from a single epoch in instantaneous positioning (Bock et al. 2000; Odijk 2001;

J. Paziewski $(\bowtie) \cdot$ P. Wielgosz

University of Warmia and Mazury in Olsztyn,

Oczapowskiego 2, Olsztyn, Poland

e-mail: jacek.paziewski@uwm.edu.pl
Wielgosz et al. 2005; Genrich and Bock 2006). Recent research concerns the evaluation of rover observations as active nodes of a ground-based augmentation systems (GBAS) network (Zinas et al. 2012), application of new signals from the Galileo system (Odijk et al. 2010, 2012), special conditions between multiple rover receivers (Giorgi et al. 2012), and development and modifications of ambiguity resolution methods (Chang et al. 2005; Cellmer et al. 2010).

The modernization of the GPS system will result in an increased number of transmitted signals and frequencies, such as L1, L2, and L5. The Galileo system will offer a number of signals transmitted on frequencies E1, E5a, E5b, E5(E5a + E5b), and E6. Application of more than two frequencies can be beneficial for ionosphere modeling, which is crucial for the ambiguity resolution. Two overlapping frequencies (1 $575.420 \mathrm{MHz}$ for L1/GPS and E1/ Galileo, and 1 176.450 MHz for L5/GPS and E5a/Galileo) will allow creating double-differenced observations between the both systems. This will result in tightly combined processing, taking into account time, coordinate system differences, and receiver inter-system biases (Odijk et al. 2012).

It is expected that the introduction of multi-frequency observations from modernized GPS and forthcoming Galileo, as well as application of tightly combined GPS + Galileo observational model, will lead to an increase in accuracy and reliability of positioning. This will also allow shortening of the observing session and extending the distance between the user receiver and reference network stations (Verhagen 2002; Julien et al. 2004; Odijk et al. 2012). Tiberius et al. (2002) showed on the basis of theoretical studies, that it would be possible to obtain 0.99999999 confidence of the ambiguity resolution with two GNSS constellations. Ji et al. (2007) investigated 
potential benefits for the ambiguity resolution with new frequency combinations formed on the basis of the new signals from the Galileo system. Zhao et al. (2005) proved that using integrated GPS + Galileo has an advantage over a single system in terms of accuracy, availability, and reliability. Recent research demonstrated that combined processing of GPS + GIOVE resulted in advancement in ambiguity resolution success rate (Odijk and Teunissen 2012)

We investigate the performance of single-epoch precise positioning with multi-frequency Galileo as well as dualfrequency GPS + Galileo observations in a tightly combined observational model. Precise single-epoch positioning is particularly vulnerable to the number of received signals and their quality. A reliable ambiguity resolution based on single-epoch data, in comparison with the on-thefly approach, is an extremely difficult and challenging task due to the low number of observations and the lack of change in satellite geometry (Hu et al. 2005, Cellmer et al. 2010; Paziewski et al. 2013). Thus, current positioning algorithms use ionospheric and tropospheric corrections derived from reference networks. Instantaneous solution is resistant to cycle slips or data gaps; it does not require any initialization or re-initialization (Bock et al. 2000), and errors or biases from previous epochs do not influence on the further epochs, i.e., all solutions are independent. The numerical tests presented are based on simulated GNSS observational data (hardware simulator) and in-housedeveloped post-processing software-GINPOS (GNSS instantaneous positioning software) (Paziewski 2012).

\section{Methodology}

Principles of precise positioning relay on double-differenced (DD) carrier phase and pseudo-range observations collected by two receivers. However, double differencing of the observations may be insufficient for error mitigation in baselines with length exceeding $\sim 10 \mathrm{~km}$ (Rizos 2002). This is due to spatial de-correlation of differential tropospheric, ionospheric, orbital, and clock errors with growing distance between the user and reference stations. An effective method, developed to overcome this issue, is the application of GNSS reference network-derived corrections. Also, in contrast to the single-baseline solution, where accuracy of the solution decreases with the baseline length, a multi-baseline network approach offers solutions almost independent of the distance between the user and the reference station network. Multi-baseline positioning with external ionospheric and geometric corrections can be regarded an extremely effective method of positioning in terms of accuracy, reliability, and session length. GBAS systems that support satellite positioning are based on this concept and are widely used (Hu et al. 2005; Bosy et al. 2007; Kashani et al. 2008).

Research studies were conducted on mitigating ionospheric delays in precise positioning. The results indicate that one of the most effective methods is to apply the external ionospheric corrections together with the estimation of residual double-differenced ionospheric delays. This method is often called ionosphere-weighted model (Teunissen 1997; Odijk 2000; Julien et al. 2004; Wielgosz 2010).

The overall procedure for positioning methodology as applied here consists of three steps: (1) processing the reference network GNSS data to derive the network corrections, (2) interpolation of ionospheric and tropospheric corrections for the user location, and (3) user solution with application of the network-derived corrections. Below, we present a brief description of the methodology developed to determine the ionospheric and tropospheric corrections from the network using multi-GNSS data (step 1).

After the ambiguities in the reference network have been resolved, the DD ionospheric delays can be accurately calculated using geometry-free (GF) linear combination of carrier phase data collected at any two frequencies (Schaer 1999):

$$
\begin{aligned}
\varphi_{k l, \mathrm{GF}}^{i j} & =\lambda_{1} \varphi_{k l, 1}^{i j}-\lambda_{2} \varphi_{k l, 2}^{i j} \\
& =-\left(1-\frac{f_{1}^{2}}{f_{2}^{2}}\right) I_{k l}^{i j}+\lambda_{1} N_{k l, 1}^{i j}-\lambda_{2} N_{k l, 2}^{i j}
\end{aligned}
$$

where the superscripts $i$ and $j$, and the subscripts $k$ and $l$ identify the satellites and stations, respectively. The function $\varphi_{\mathrm{GF}}$ is the geometry-free DD carrier phase observable; $\varphi_{1}$, and $\varphi_{2}$ are the DD carrier phase observables; $\lambda_{1}$ and $\lambda_{2}$ are the applied wavelengths; $f_{1}$ and $f_{2}$ are the applied frequencies; $I$ is the DD ionospheric delay; and $N_{1}$ and $N_{2}$ are the DD carrier phase ambiguities.

In the presented research, the GPS + Galileo L1/E1 and L5/E5a signals were applied. DD ionospheric delays were computed for mixed GPS and Galileo signals for each independent baseline in the reference network solution (step 1). In order to apply the ionospheric corrections in the rover solution, the DD ionospheric delays from the reference network solution were decomposed to undifferenced (UD) delays and interpolated to the user receiver approximate location (step 2). The decomposition procedure is based on least-squares estimation with constraints. It is used for the determination of biased undifferenced ionospheric delays from the network-derived DD delays. In this step, additional pseudo-observations with low weights were introduced to the system in order to overcome the rank deficiency inherited into decomposition of DD observables into UD ones. The pseudo-observations do not influence on the adjustment because of their low weights. 
However, the resulting UD ionospheric delays are biased. At the step 3 (rover solution), the interpolated UD ionospheric delays were again converted to DD delays which serve as weighted corrections (Wielgosz et al. 2005). Note that, UD biases are removed when DD ionospheric corrections are calculated (due to differencing of the UD corrections).

As our previous research has shown for precise positioning with very short sessions, it is not recommended to estimate zenith tropospheric delay (ZTD) in the rover solution due to the slight change in satellite geometry (Wielgosz et al. 2011a). For reliable estimation of ZTD, a significant distance between the stations is required (Dach et al. 2007; Wielgosz et al. 2011b). Therefore, the best results were obtained introducing external, networkderived tropospheric corrections for the rover. These corrections are derived in the reference network solution when the tropospheric ZTDs are estimated at reference stations. These are later used for calculating the ZTD at the rover position. The ZTDs estimated at the reference stations are reduced to the mean sea level using modified Hopfield troposphere model and then interpolated spatially to the rover position and temporarily to the time of the particular epoch. Finally, the interpolated ZTDs at the mean sea level are converted to the height of the rover using again the modified Hopfield model, resulting in ZTD corrections at the rover (Wielgosz et al. 2011c).

The generalized multi-frequency and multi-system model of observation equations for precise positioning using DD carrier phase and pseudo-range observations is presented below (Eqs. 2, 3). The model requires at least dual-frequency data. The equation is given for a single epoch and a particular double difference of observations from satellites $i$ and $j$ at stations $k$ and $l$ and frequency $n$, and is applied for each of the used frequency:

$$
\begin{aligned}
& \lambda_{n} \varphi_{k l, n}^{i j}-\varrho_{k l}^{i j}-\left(\alpha_{k}^{i} \mathrm{ZTD}_{k}-\alpha_{k}^{j} \mathrm{ZTD}_{k}-\alpha_{l}^{i} \mathrm{ZTD}_{l}+\alpha_{l}^{j} \mathrm{ZTD}_{l}\right) \\
& \quad+I_{k l}^{i j} \frac{f_{1}^{2}}{f_{n}^{2}}-\lambda_{n} N_{k l, n}^{i j}=0 \\
& P_{k l, n}^{i j}-\varrho_{k l}^{i j}-\left(\alpha_{k}^{i} \mathrm{ZTD}_{k}-\alpha_{k}^{j} \mathrm{ZTD}_{k}-\alpha_{l}^{i} \mathrm{ZTD}_{l}+\alpha_{l}^{j} \mathrm{ZTD}_{l}\right) \\
& \quad-I_{k l}^{i j} \frac{f_{1}^{2}}{f_{n}^{2}}=0
\end{aligned}
$$

where $\varrho$ is the DD geometric distance, $\alpha$ is the troposphere mapping function coefficient, ZTD is the tropospheric zenith total delay, $P$ is the DD pseudo-range, and $f_{1}$ denotes the first used frequency.

The mathematical model is applied for both reference and user solutions. The model is filtered using the leastsquares adjustment with a priori parameter constraints (Leick 2004; Xu 2007). The applied observational model assumes modeling several parameters such as reference station and rover coordinates, DD ambiguities, and ZTDs. For every DD epoch, a new DD ionospheric delay parameter is introduced. Introduction of the DD ionospheric delays into the state vector causes the degrees of freedom of the filter to decrease. In order to increase the observational model redundancy, stochastic constraints are applied. The constraint equations are treated in the model as pseudo-observations with weights computed from the a priori variance-covariance estimate. In the algorithm, the a priori reference and rover stations coordinates, ZTDs, and DD ionospheric delays are stochastically constrained. Thus, the total observational model consist of two groups of observation equations: linearized observational equations with the respective design matrix, observed minus computed vector, and weight matrix $\left(A, L, P_{L}\right.$, respectively), and the pseudo-observation equations with their design matrix, observed minus computed vector, and weight matrix ( $B, W, P_{W}$, respectively). Combining both the equation groups, the adjustment model can expressed as follows (Xu 2007):

$$
\left.\begin{array}{l}
V=A d_{X}-L, P_{L} \\
U=B d_{X}-W, P_{W}
\end{array}\right\}
$$

$V$ is the vector of residuals of linearized observations, and $U$ is the vector of residuals of constraints observations. The adjusted parameters are computed as the sum of the a priori values of the parameters $\left(X^{0}\right)$ and the adjusted corrections $\left(d_{X}\right)$, i.e., $X=X^{0}+d_{X}$.

The combined least-squares solution is

$$
\left(A^{T} P_{L} A+B^{T} P_{W} B\right) d_{X}-\left(A^{T} P_{L} L+B^{T} P_{W} W\right)=0
$$

The solution (5) further implies that the two types of observation are stochastically independent.

When GPS + Galileo observations are processed together, a single reference satellite is used. This model is called as tightly combined (integrated). Research shows that when processing combined GPS + Galileo DD observations that were collected with different receiver types, the mathematical model should take into account phase and pseudo-range inter-system biases (ISB) (Odijk et al. 2012). The ISB is the difference between the receiver hardware delays of signals of two systems (Hegarty et al. 2004). Another possibility is to correct observations with known ISB (Odijk and Teunissen 2012). In the presented research, all observations were collected with the same Septentrio TUR-N receiver; thus, ISB was absent in the observational model.

The data processing using the presented methodology was performed in the instantaneous mode, i.e., each epoch was processed independently. For the ambiguity resolution, the LAMBDA method was applied (Teunissen 1995). Validation of the solution was performed using W-ratio test (Wang et al. 1998). 


\section{GNSS data simulation}

The experiments presented below rely on post-processing of GNSS signals in various configurations, such as using a single-system (Galileo or GPS) or a multi-system observations with different number of frequencies. We assume that the full Galileo constellation and the modernized GPS with L5 signals are available for all satellites. Since the Galileo system is not fully operational, and the modernization of the GPS system has not yet been completed, the observational data were generated by a hardware GNSS signal simulator. We used the SPIRENT GSS7700/7800 hardware signal simulator and the Septentrio TUR-N GNSS receiver at the ESTEC/ESA center.

The simulated session lasted almost $3.5 \mathrm{~h}$, starting at 11:30 UTC and ending at 14:55 UTC of July 1, 2011. The signals were simulated for four sites. Three of these are existing reference stations of the Polish multifunctional GBAS network (ASG-EUPOS). These stations, TORU, LESZ, and RWMZ, served as the reference stations in postprocessing tests. The last simulated station, KK16, served as the static user receiver. The average separation between the reference stations is approximately $200 \mathrm{~km}$. The baselines connecting reference stations and rover ranged from 99 to $148 \mathrm{~km}$ (Fig. 1).

The simulated signals from the SPIRENT simulator were free of ionospheric and tropospheric refraction. This allowed for introduction of tropospheric and ionospheric delays from external sources. In order to introduce tropospheric delays into the simulated observations, ZTD from the official ASG-EUPOS solution was used (Szafranek et al. 2013). The ZTD values were interpolated spatially and temporarily to the simulated station locations, and then mapped into slant delays using GMF mapping function (Boehm et al. 2006). Next, the obtained slant tropospheric delays were added to the simulated carrier phase and pseudo-range observations. The ionospheric slant delays, added later to the "clean" simulated data, were computed

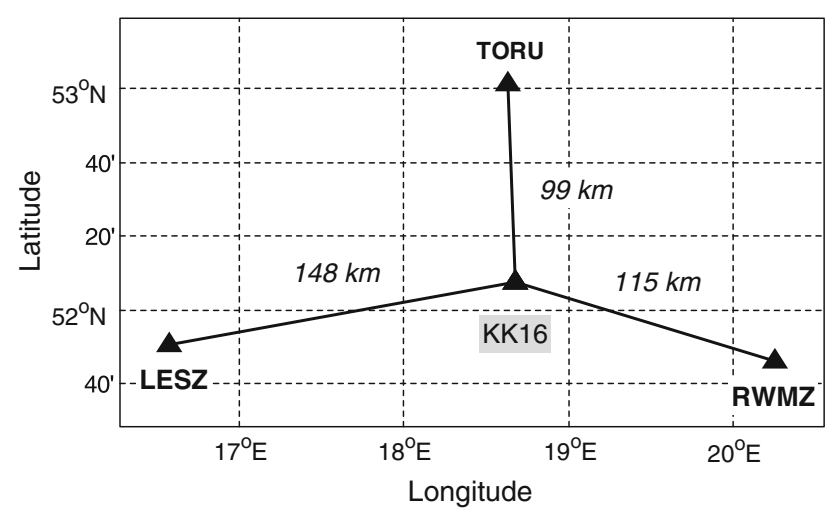

Fig. 1 Experimental network and baselines used in the rover solution from global ionosphere maps (GIM) in IONEX format obtained from the Global Assimilative Ionospheric Model (GAIM) model developed by Jet Propulsion Laboratory (JPL) (Mandrake et al. 2005). It should be noted that JPL maps have spatial and temporal resolution of $1^{\circ} \times 1^{\circ} \times 15 \mathrm{~min}$. During the analyzed period, the ionosphere was active, but not stormy, with maximum of geomagnetic index $K p$ of $3+$.

\section{Performance of the single-epoch ambiguity resolution and positioning}

Mean coordinate residuals $(\mathrm{d} N, \mathrm{~d} E$, and $\mathrm{d} U$ ) with their respective standard deviations (STD) were analyzed in order to evaluate the quality of the solutions. The performance of the ambiguity resolution was analyzed as the ratio of the number of epochs with all correctly solved and validated ambiguities to the number of all processed epochs; this is called the ambiguity resolution and validation success rate (ASR). Note that in each epoch, the ambiguities are treated independently as new and resolved. The ambiguity validation failure rate (AFR) shows the ratio of the number of epochs with incorrectly resolved ambiguities which passed the ambiguity validation procedure (false fixes) to the number of all processed epochs. Of course, the position obtained with wrong ambiguities has large errors.

\section{Processing strategies}

The processing strategies use dual-, triple-, or quadruplefrequency carrier phase and pseudo-range observations. The LAMBDA method was used, with the W-ratio threshold set to 2.5 , for the resolution and validation of the DD ambiguities. The tropospheric refraction was mitigated with the application of tropospheric ZTDs interpolated from the reference network solution to the user location. Since a single-epoch solution was applied, these interpolated ZTDs were tightly constrained with $\sigma_{\mathrm{ZTD}}=0.0003 \mathrm{~m}$ in the adjustment. As it was mentioned above, mitigation of the ionospheric refraction assumed introducing interpolated ionospheric corrections from the reference network solution with the estimation of the residual DD ionospheric delays with a priori sigma $\sigma_{\mathrm{ION}}=0.25 \mathrm{~m}$. This value was set on the basis of empirical studies (Wielgosz et al. 2005). It corresponds to the precision of network-derived ionospheric corrections (Fig. 3), which depend on the baseline length and the ionospheric activity (Christie et al. 1998). The broadcast ephemerides were used to compute satellite positions. For each station, about $3.5 \mathrm{~h}$ of GNSS data were divided into 410 independent single-epoch sessions with $30 \mathrm{~s}$ interval. 
Five processing strategies employing different combinations of GNSS signals and systems were tested, namely (1) GPS L1\&L5; (2) Galileo E1\&E5a; (3) Galileo E1\&E5a\&E6; (4) Galileo E1\&E5a\&E5b\&E6; and (6) tightly combined GPS + Galileo L1/E1\&L5/E5a. This selection allowed for testing the performances of GPS versus Galileo when using signals from the same frequencies (Strategies 1 and 2), dual-frequency Galileo solutions versus triple- and quadruple-frequency solutions (Strategies 2, 3, and 4), or single-systems solutions versus multi-system solutions (Strategies 1, 2, and 5).

\section{Processing cases}

The performance of the instantaneous multi-frequency, multi-system positioning was analyzed for single baseline (99 km baseline, TORU-KK16) and a network solution (99-148 km baselines connecting KK16 with TORU, LESZ, and RWMZ reference stations). In all cases, network-derived atmospheric corrections were applied. In addition, all calculations were performed for clear-sky satellite visibility $\left(15^{\circ}\right.$ elevation mask) and obstructed satellite visibility $\left(30^{\circ}\right.$ elevation mask). In the clear-sky case, $15^{\circ}$ mask reflects regular settings in the GNSS rover receivers. On the other hand, $30^{\circ}$ mask simulates adverse conditions with obstructed visibility (e.g., trees, urban canyons).

Figure 2 presents the number of the satellites for each system observed at the user location with $15^{\circ}$ (top panel) and $30^{\circ}$ (bottom panel) elevation mask. In case of the $15^{\circ}$ elevation mask, the number of observed satellites for both systems varied from 11 to 17 . Simulating signal obstructions with $30^{\circ}$ elevation mask, the number of the observed satellites decreased substantially to 6-12. In this case, the number of the satellites tracked by each system separately never exceeded 6, sometimes even dropping to 2 (Fig. 2, bottom panel). In such a situation, a single satellite system cannot provide a solution for the position.

\section{Ionospheric corrections}

Figure 3 (top panel) presents DD ionospheric delays observed over the baselines of the network (Fig. 1). Different colors in the figure correspond to different DD observable. The values can be regarded as true since they were derived from JPL GAIM model and introduced into the simulated data. The figure shows that most of the DD ionospheric delays are within the range $\pm 0.40 \mathrm{~m}$. In the processing, these delays are corrected by the reference network-derived corrections. In general, it is desirable that the true error of the corrections does not exceed $10 \mathrm{~cm}$, which is equivalent to half of the L1 cycle. However, in the ionosphere-weighted model, this error can be larger. Since the observations from the reference stations and from the
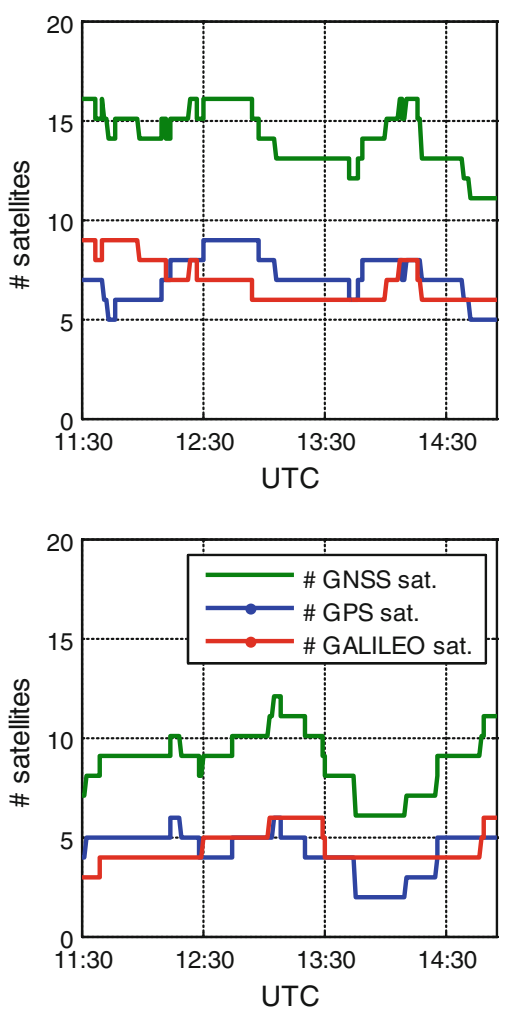

Fig. 2 Number of observed satellites at user station KK16 with $15^{\circ}$ (top) and $30^{\circ}$ elevation mask (bottom)
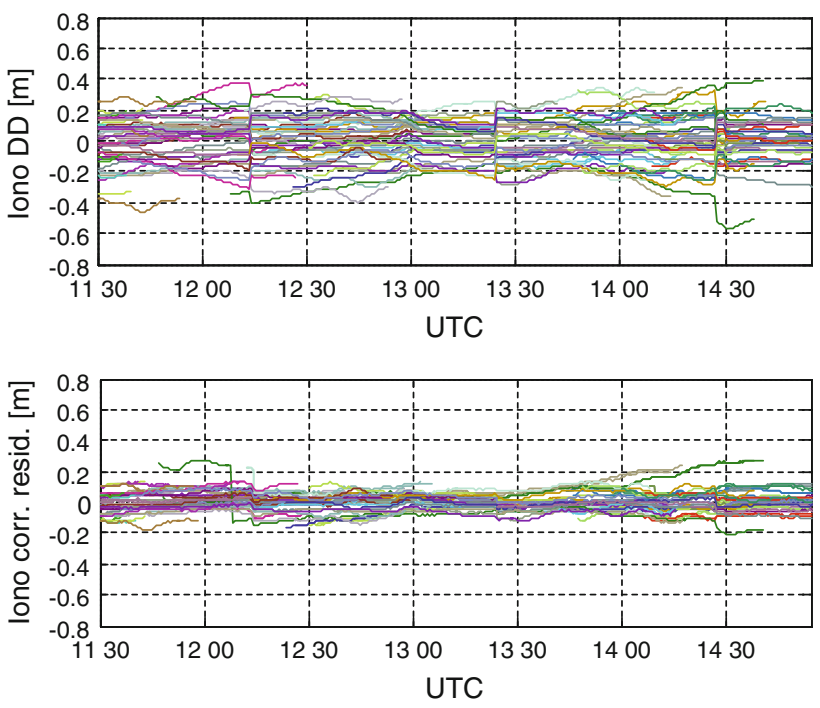

Fig. 3 Double-differenced ionospheric delays over the baselines at the network (top) and network-derived DD ionospheric correction residuals (bottom)

user receiver are subject to known ionospheric delays derived from JPL GAIM model, it is possible to calculate real accuracy of the corrections by, e.g., comparing the network-derived DD ionospheric corrections with the model values. The bottom panel (Fig. 3) shows the 
differences between the network-derived DD ionospheric corrections and the true ionospheric delays for the baselines of the network. These residuals represent accuracy of the interpolated ionospheric corrections, whose accuracy depends mostly on the spatial correlation of the ionosphere. Application of the network-derived ionospheric corrections clearly reduces ionospheric biases in the system and hence improves the quality of the float ambiguities. This in turn improves ambiguity fixing.

Results with $15^{\circ}$ elevation mask

Below are results of instantaneous single- and multi-baseline rover solutions using an elevation angle of $15^{\circ}$. One may expect that in this case, adding Galileo satellites will bring minor improvement since there are usually enough GPS satellites to provide precise position.

\section{Single-baseline solution}

The results refer to the single-baseline solution TORUKK16 of $99 \mathrm{~km}$ obtained with $15^{\circ}$ elevation mask. Singlebaseline solution for long baselines clearly demonstrates the challenges for single-epoch positioning. For such a long baseline, the de-correlation of the ionosphere results in lower-quality DD ionospheric corrections. This in turn makes fast ambiguity resolution much more difficult.

The resulting ambiguity resolution and validation success rate (ASR) varied from only 57.8 up to $97.6 \%$
(Table 1). The best results regarding the ambiguity resolution were obtained for a strategy assuming combined processing of GPS and Galileo signals (Strategy 5). Also, for this strategy, the lowest number of incorrect solutions was obtained (AFR $=0.0 \%)$. The repeatability of the coordinates is based on correct fixed solutions only, and it was comparable for all the strategies. Standard deviations for the horizontal components did not exceed $3 \mathrm{~mm}$. At the same time, standard deviations for the height component were lower than $7 \mathrm{~mm}$ (Table 1).

It is also observed that in case of Galileo-only solutions (Strategies 2, 3, and 4), a higher number of the applied frequencies resulted in more reliable ambiguity resolution (higher ASR and lower AFR).

\section{Multi-baseline solution}

The multi-baseline solution has advantage over the singlebaseline solution regarding both the coordinate and ambiguity resolution domains. The ASR varied from 77.1 to the $98.5 \%$ (Table 2). For example, in case of Strategy 1 (GPS L1\&L5), the ASR increased from 57.8 to $77.1 \%$ with respect to the single-baseline solution. For the Strategies $2-4$, this improvement reached approximately $21-24 \%$. Also, the multi-baseline solution confirmed its advantage in position reliability, with no validation failures.

In addition, using more frequencies caused better performance of the ambiguity resolution. Again, the best results were obtained for Strategy 5 that uses two systems

Table 1 Statistics of single-baseline solution with $15^{\circ}$ elevation mask

\begin{tabular}{|c|c|c|c|c|c|c|c|c|}
\hline \multirow[t]{2}{*}{ \# Strategy } & \multicolumn{2}{|c|}{$N(\mathrm{~cm})$} & \multicolumn{2}{|c|}{$E(\mathrm{~cm})$} & \multicolumn{2}{|c|}{$U(\mathrm{~cm})$} & \multirow{2}{*}{$\begin{array}{l}\text { ASR } \\
(\%)\end{array}$} & \multirow{2}{*}{$\begin{array}{l}\text { AFR } \\
(\%)\end{array}$} \\
\hline & $\mathrm{d} N$ & sdt_N & $\mathrm{d} E$ & $\operatorname{std} \_E$ & $\mathrm{~d} U$ & std_U & & \\
\hline 1. GPS L1\&L5 & 0.2 & 0.3 & 0.0 & 0.2 & 0.1 & 0.6 & 57.8 & 0.7 \\
\hline 2. GAL E1\&E5a & 0.1 & 0.3 & 0.0 & 0.2 & 0.0 & 0.5 & 67.1 & 4.1 \\
\hline 3. GAL E1\&E5a\&E6 & 0.1 & 0.3 & 0.0 & 0.2 & 0.1 & 0.5 & 71.5 & 2.7 \\
\hline 4. GAL E1\&E5a\&E5b\&E6 & 0.1 & 0.3 & 0.0 & 0.2 & 0.0 & 0.5 & 73.4 & 3.4 \\
\hline 5. GPS L1\&L5 GAL E1\&E5a & 0.0 & 0.3 & 0.0 & 0.2 & 0.1 & 0.7 & 97.6 & 0.0 \\
\hline
\end{tabular}

The baseline length is $99 \mathrm{~km}$

Table 2 Statistics of the multi-baseline solution with $15^{\circ}$ elevation mask

\begin{tabular}{|c|c|c|c|c|c|c|c|c|}
\hline \multirow[t]{2}{*}{ \# Strategy } & \multicolumn{2}{|c|}{$N(\mathrm{~cm})$} & \multicolumn{2}{|c|}{$E(\mathrm{~cm})$} & \multicolumn{2}{|c|}{$U(\mathrm{~cm})$} & \multirow{2}{*}{$\begin{array}{l}\text { ASR } \\
(\%)\end{array}$} & \multirow{2}{*}{$\begin{array}{l}\text { AFR } \\
(\%)\end{array}$} \\
\hline & $\mathrm{d} N$ & sdt_N & $\mathrm{d} E$ & std_E & $\mathrm{d} U$ & std_U & & \\
\hline 1. GPS L1\&L5 & 0.1 & 0.3 & 0.0 & 0.1 & 0.0 & 0.5 & 77.1 & 0.0 \\
\hline 2. GAL E1\&E5a & 0.1 & 0.2 & 0.0 & 0.1 & 0.0 & 0.4 & 81.2 & 0.0 \\
\hline 3. GAL E1\&E5a\&E6 & 0.1 & 0.2 & 0.0 & 0.1 & 0.0 & 0.4 & 88.3 & 0.0 \\
\hline 4. GAL E1\&E5a\&E5b\&E6 & 0.1 & 0.2 & 0.0 & 0.1 & 0.0 & 0.4 & 91.0 & 0.0 \\
\hline 5. GPS L1\&L5 GAL E1\&E5a & 0.0 & 0.2 & 0.2 & 0.2 & 0.1 & 0.7 & 98.5 & 0.0 \\
\hline
\end{tabular}


and two signal frequencies (ASR $=98.5 \%$ ). Also, a very high ASR was obtained for Strategy 4 with four Galileo frequencies E1\&E5a\&E5b\&E6 (91.0\%).

Results with $30^{\circ}$ elevation mask

The results refer to instantaneous single- and multi-baseline rover solutions in case of application of $30^{\circ}$ elevation mask. One may expect that in this case, adding Galileo satellites will bring noticeable improvement as there may be not enough GPS satellites to provide precise position.

\section{Single-baseline solution}

The most challenging test for the presented methodology was, however, processing long single-baseline with limited satellite visibility. A low number of satellites when processing each system separately and high residual ionospheric delays caused poor performance of the ambiguity resolution (Strategies 1-4, Table 3). On the other hand, this case allowed for better assessment of the impact of the combined processing of GPS + Galileo observations. The resulting ASR for Strategy 5 (GPS + Galileo) amounted to $92.7 \%$, which is more than 9 times higher in comparison with Strategy 1 (GPS L1\&L5) and almost 3 times higher in comparison with Strategy 2 (Galileo E1\&E5a). Also, the reliability of the ambiguity resolution in case of the multisystem solution was very high with the AFR reaching only $0.2 \%$, even though this is only a single-baseline solution.

\section{Multi-baseline solution}

As expected from the results presented in the sections above, multi-baseline solution provides the best results in all strategies and situations. It brings improvement in the ambiguity resolution for all the strategies (Table 4). However, despite limited satellite visibility, acceptable results are obtained in case of the multi-system solution (Strategy 5). This strategy still allowed to correctly solving ambiguities $99 \%$ of the epochs with no false fixes. This also shows that in real-life conditions, when the user may expect signal obstructions, only collecting and processing the data from both GPS and Galileo systems allows accurate and reliable instantaneous positioning with high success.

Figure 4 summarizes the ambiguity resolution and validation success rate (ASR) obtained in analyzed situations. Noticeable differences between strategies are observed. Introduction of additional signals/frequencies in Galileo system results in more successful ambiguity resolution. Also, the combined GPS + Galileo solution has a clear advantage over any single-system solution. It is clearly visible that the tightly combined GPS + Galileo solution provides the highest values of the ASR parameter. In case of signal obstructions and a low number of the observed satellites, introducing observations from additional GNSS system (Strategy 5) has the greatest impact on the instantaneous ambiguity resolution and positioning results.

Table 3 Statistics of the single-baseline solution with $30^{\circ}$ elevation mask

\begin{tabular}{|c|c|c|c|c|c|c|c|c|}
\hline \multirow[t]{2}{*}{ \# Strategy } & \multicolumn{2}{|c|}{$N(\mathrm{~cm})$} & \multicolumn{2}{|c|}{$E(\mathrm{~cm})$} & \multicolumn{2}{|c|}{$U(\mathrm{~cm})$} & \multirow{2}{*}{$\begin{array}{l}\text { ASR } \\
(\%)\end{array}$} & \multirow{2}{*}{$\begin{array}{l}\text { AFR } \\
(\%)\end{array}$} \\
\hline & $\mathrm{d} N$ & sdt_N & $\mathrm{d} E$ & std_E & $\mathrm{d} U$ & std_U & & \\
\hline 1. GPS L1\&L5 & 0.0 & 0.8 & -0.1 & 0.4 & -0.3 & 1.0 & 10.0 & 3.2 \\
\hline 2. GAL E1\&E5a & 0.1 & 0.4 & 0.0 & 0.4 & -0.4 & 2.9 & 31.2 & 12.2 \\
\hline 3. GAL E1\&E5a\&E6 & 0.1 & 0.4 & 0.0 & 0.4 & -0.6 & 3.6 & 34.1 & 12.7 \\
\hline 4. GAL E1\&E5a\&E5b\&E6 & 0.1 & 0.4 & 0.0 & 0.4 & -0.4 & 3.1 & 36.3 & 13.7 \\
\hline 5. GPS L1\&L5 GAL E1\&E5a & 0.0 & 0.5 & -0.1 & 0.3 & -0.3 & 1.4 & 92.7 & 0.2 \\
\hline
\end{tabular}

The baseline length is $99 \mathrm{~km}$

Table 4 Statistics of the multi-baseline solution with $30^{\circ}$ elevation mask

\begin{tabular}{|c|c|c|c|c|c|c|c|c|}
\hline \multirow[t]{2}{*}{ \# Strategy } & \multicolumn{2}{|c|}{$N(\mathrm{~cm})$} & \multicolumn{2}{|c|}{$E(\mathrm{~cm})$} & \multicolumn{2}{|c|}{$U(\mathrm{~cm})$} & \multirow{2}{*}{$\begin{array}{l}\text { ASR } \\
(\%)\end{array}$} & \multirow{2}{*}{$\begin{array}{l}\text { AFR } \\
(\%)\end{array}$} \\
\hline & $\mathrm{d} N$ & sdt_N & $\mathrm{d} E$ & std_E & $\mathrm{d} U$ & std_U & & \\
\hline 1. GPS L1\&L5 & 0.0 & 0.6 & 0.0 & 0.3 & -0.2 & 0.8 & 14.4 & 2.0 \\
\hline 2. GAL E1\&E5a & 0.1 & 0.3 & 0.0 & 0.3 & -0.4 & 2.9 & 39.0 & 1.5 \\
\hline 3. GAL E1\&E5a\&E6 & 0.1 & 0.3 & 0.0 & 0.3 & -0.2 & 3.0 & 50.0 & 3.2 \\
\hline 4. GAL E1\&E5a\&E5b\&E6 & 0.1 & 0.3 & 0.0 & 0.3 & -0.1 & 2.8 & 53.9 & 3.4 \\
\hline 5. GPS L1\&L5 GAL E1\&E5a & -0.3 & 0.5 & -0.4 & 0.3 & 0.2 & 1.8 & 99.0 & 0.0 \\
\hline
\end{tabular}




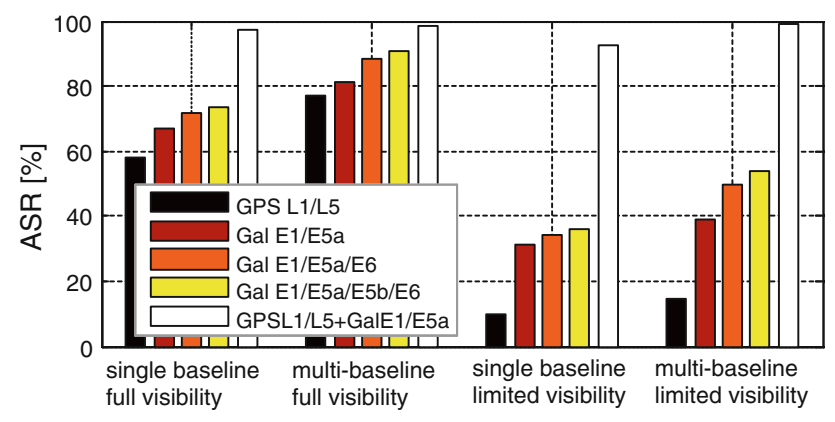

Fig. 4 Ambiguity resolution and validation success rates for analyzed strategies

\section{Summary and conclusion}

Several processing strategies based on processing of dualfrequency GPS-only signals, dual-to quadruple-frequency Galileo-only signals, and tightly combined processing of GPS + Galileo signals were tested and analyzed for their applicability for long-range precise instantaneous positioning. The best results were always obtained with combined processing of GPS + Galileo signals in a multi-station solution. This solution provides the highest instantaneous ambiguity resolution success rate with no false fixes in good observing conditions and with very rare false fixes in adverse conditions.

It has also been confirmed that the application of multifrequency observations in case of Galileo system has advantage over dual-frequency solution, especially considering long baselines and single-baseline processing.

Note that, this research is based on the simulated data, and even though the observations came from a hardware GNSS signal simulator and were collected by standard GNSS receivers, they may still present better quality than the data collected under real-field conditions. However, our results confirm the great utility of the Galileo constellation and advantages of a multi-system solution, and also point out to directions of further studies. It may be also concluded that even in case of a limited number of Galileo satellites in the near future, their signals will surely improve the availability and quality of the satellite precise positioning when combined with GPS data.

Finally, it can be concluded that tightly combined GPS + Galileo positioning supported with networkderived ionospheric and tropospheric corrections provides accurate and reliable solution even when processing observations from just a single observational epoch.

Acknowledgments The authors would like to express their gratitude to Jaron Samson from ESTEC/ESA for an opportunity to collect data with GNSS signal simulator. This research was supported by a grant agreed by the decision DEC-2011/03/N/ST10/05317 from Polish National Center of Science and National Centre for Research and Development, Grant No. NR09-0010-10/2010.
Open Access This article is distributed under the terms of the Creative Commons Attribution License which permits any use, distribution, and reproduction in any medium, provided the original author(s) and the source are credited.

\section{References}

Bock Y, Nikolaidis R, de Jonge PJ, Bevis M (2000) Instantaneous geodetic positioning at medium distances with the Global Positioning System. J Geophys Res 105(B12):28233-28253

Boehm J, Niell A, Tregoning P, Schuh H (2006) Global mapping function (GMF): a new empirical mapping function based on numerical weather model data. Geophys Res Lett 33(7):L07304. doi: $10.1029 / 2005$ GL025546

Bosy J, Graszka W, Leonczyk M (2007) ASG-EUPOS—a multifunctional precise satellite positioning system in Poland. Eur J Nav $5(4): 2-6$

Cellmer S, Wielgosz P, Rzepecka Z (2010) Modified ambiguity function approach for GPS carrier phase positioning. J Geod 84(4):267-275. doi:10.1007/s00190-009-0364-8

Chang X-W, Yang X, Zhou T (2005) MLAMBDA: a modified LAMBDA method for integer least-squares estimation. J Geod 79(9):552-565. doi:10.1007/s00190-005-0004-х

Christie J, Pervan B, Enge P, Powell J, Parkinson B, Ko P (1998) Analytical and experimental observations of ionospheric and tropospheric decorrelation effects for differential satellite navigation during precision approach. In: Proceedings of the ION-GPS1998, Institute of Navigation, Nashville, Tennessee, pp 739-748

Dach R, Hugentobler U, Fridez P, Meindl M (2007) Bernese GPS software version 5.0. Astronomical Institute University of Bern, Bern

Genrich JF, Bock Y (2006) Instantaneous geodetic positioning with 10-50 Hz GPS measurements: noise characteristics and implications for monitoring networks. J Geophys Res 111:B03403. doi: $10.1029 / 2005 J B 003617$

Giorgi G, Teunissen PJG, Gourlay TP (2012) Instantaneous Global Navigation Satellite System (GNSS) based attitude determination for maritime applications. IEEE J Ocean Eng 37(3):348-362. doi:10.1109/JOE.2012.2191996

Hegarty C, Powers E, Fonville B (2004) Accounting for timing biases between GPS, modernized GPS, and Galileo signals. In: Proceedings of the 36th annual precise time and time interval (PTTI) Meeting Washington, DC, 7-9 December, pp 307-317

Hu G, Abbey DA, Castleden WE, Earls C, Ovstedal O, Weihing D (2005) An approach for instantaneous ambiguity resolution for medium- to long-range multiple reference station networks. GPS Solut 9(1):1-11. doi:10.1007/s10291-004-0120-8

Ji S, Chen W, Zhao C, Ding X, Chen Y (2007) Single epoch ambiguity resolution for Galileo with the CAR and LAMBDA methods. GPS Solut 11(4):259-268. doi:10.1007/s10291-0070057-9

Julien O, Alves P, Cannon ME, Lachapelle G (2004) Improved triplefrequency GPS/Galileo carrier phase ambiguity resolution using a stochastic ionosphere modeling. In: Proceedings of the IONNTM-2004, Institute of Navigation, San Diego, CA, pp 441-452

Kashani I, Wielgosz P, Grejner-Brzezinska DA, Mader GL (2008) A new network-based rapid-static module for the NGS online positioning user service-OPUS-RS. Navigation 55(3):255-264

Leick A (2004) GPS satellite surveying, 3rd edn. Wiley, New Jersey

Mandrake L, Wilson B, Wang C, Hajj G, Mannucci A, Pi X (2005) A performance evaluation of the operational Jet Propulsion Laboratory/University of Southern California Global Assimilation Ionospheric Model (JPL/USC GAIM). J Geophys Res 110:A12306 
Odijk D (2000) Stochastic modelling of the ionosphere for fast GPS ambiguity resolution. In: Proceedings of the Geodesy beyond 2000 - the challenges of the first decade, IAG General Assembly, Birmingham, UK, July 19-30, vol 121, pp 387-392. doi:10. 1007/978-3-642-59742-8_63

Odijk D (2001) Instantaneous precise GPS positioning under geomagnetic storm conditions. GPS Solut 5(2):29-42. doi:10. 1007/PL00012884

Odijk D, Teunissen PJG (2012) Characterization of between-receiver GPS-Galileo inter-system biases and their effect on mixed ambiguity resolution. GPS Solut 17(4):521-533. doi:10.1007/ s10291-012-0298-0

Odijk D, Verhagen S, Teunissen PJG, Hernández-Pajares M, Juan JM, Sanz J, Samson J, Tossaint M (2010) LAMBDA-based ambiguity resolution for next-generation GNSS wide area RTK. IONNTM-2010. Institute of Navigation, San Diego, CA, pp 566-576

Odijk D, Teunissen PJG, Huisman L (2012) First results of mixed GPS + GIOVE single-frequency RTK in Australia. J Spat Sci 57(1):3-18. doi:10.1080/14498596.2012.679247

Paziewski J (2012) New algorithms for precise positioning with use of Galileo and EGNOS European satellite navigation systems. $\mathrm{PhD}$ Dissertation, University of Warmia and Mazury in Olsztyn (in Polish)

Paziewski J, Wielgosz P, Krukowska M (2013) Application of SBAS pseudorange and carries phase signals to precise instantaneous single-frequency positioning. Acta Geodyn Geomater 10(4):421-430. doi:10.13168/AGG.2013.0041

Rizos C (2002) Network RTK research and implementation: a geodetic perspective. J Glob Position Syst 2(1):144-150

Schaer S (1999) Mapping and predicting Earth's Ionosphere using Global Positioning System, PhD Dissertation, Astronomical Institute, University of Berne, Switzerland

Szafranek K, Bogusz J, Figurski M (2013) GNSS reference solution for permanent station stability monitoring and geodynamical investigations: the ASG-EUPOS case study. Acta Geodyn Geomater 10(1):67-75

Teunissen PJG (1995) The least-squares ambiguity decorrelation adjustment: a method for fast GPS integer ambiguity estimation. J Geod 70(1-2):65-82. doi:10.1007/BF00863419

Teunissen PJG (1997) The geometry-free GPS ambiguity search space with a weighted ionosphere. J Geod 71(6):370-383. doi:10.1007/s001900050105

Tiberius C, Pany T, Eissfeller B, Joosten P, Verhagen S (2002) 0.99999999 confidence ambiguity resolution with GPS and Galileo. GPS Solut 6(1-2):96-99. doi:10.1007/s10291-002-0022-6

Verhagen S (2002) Performance analysis of GPS, Galileo and Integrated GPS-Galileo. In: Proceedings of the ION-GPS-2002, Institute of Navigation, September, Portland, pp 2208-2215

Wang J, Stewart M, Tsakiri M (1998) A discrimination test procedure for ambiguity resolution on-the-fly. J Geod 72(11):644-653. doi:10.1007/s001900050204

Wielgosz P (2010) Quality assessment of GPS rapid static positioning with weighted ionospheric parameters in generalized least squares. GPS Solut 15(2):89-99. doi:10.1007/s10291-0100168-6

Wielgosz P, Kashani I, Grejner-Brzezinska DA (2005) Analysis of long-range network RTK during severe ionospheric storm. J Geod 79(9):524-531. doi:10.1007/s00190-005-0003-y

Wielgosz P, Cellmer S, Rzepecka Z, Paziewski J, Grejner-Brzezinska DA (2011a) Troposphere modeling for precise GPS rapid static positioning in mountainous areas. Meas Sci Technol 22(4). doi:10.1088/0957-0233/22/4/045101

Wielgosz P, Paziewski J, Baryła R (2011b) On constraining zenith tropospheric delays in processing of local GPS networks with Bernese software. Surv Rev 43(323):472-483. doi:10.1179/ $003962611 X 13117748891877$
Wielgosz P, Paziewski J, Krankowski A, Kroszczynski K, Figurski M (2011c) Results of the application of tropospheric corrections from different troposphere models for precise GPS rapid static positioning. Acta Geophys 60(4):1236-1257. doi:10.2478/ s11600-011-0078-1

Xu G (2007) GPS: theory, algorithms and applications, 2nd edn. Springer, Berlin

Zhao C, Ou J, Yuan Y (2005) Positioning accuracy and reliability of GALILEO, integrated GPS-GALILEO system based on single positioning model. Chin Sci Bull 50(12):1252-1260. doi:10. 1007/BF03183701

Zinas N, Parkins A, Ziebart M (2012) Improved network-based single-epoch ambiguity resolution using centralized GNSS network processing. GPS Solut 17(1):17-27. doi:10.1007/ s10291-012-0256-X

\section{Author Biographies}

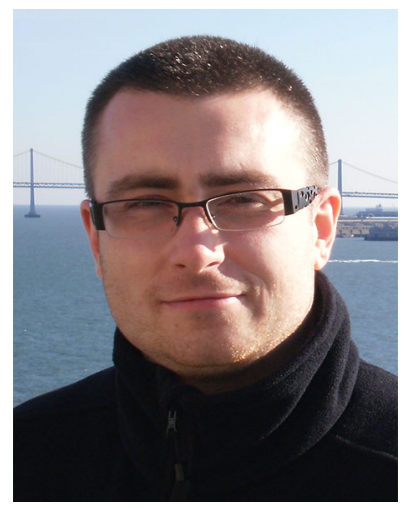

Jacek Paziewski is an Assistant Professor at the University of Warmia and Mazury (UWM) in Olsztyn. In 2012, he obtained his Ph.D. with the doctoral thesis "New algorithms for precise positioning with use of European navigation satellite systems Galileo and EGNOS." His research interests cover algorithms and software development for precise network multi-GNSS positioning, mitigation of tropospheric and ionospheric delays in GNSS positioning, and application of GNSS technology in engineering surveying.

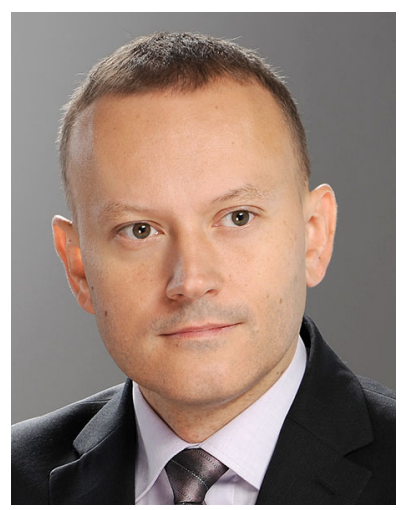

Dr. Pawel Wielgosz is an Associate Professor at the Faculty of Geodesy and Land Management of the University of Warmia and Mazury in Olsztyn (UWM), Poland, where he earned his Ph.D. in Geodesy in 2002. From 2002 to 2005, he was a Visiting Scholar at The Ohio State University (OSU). His research interests cover satellite navigation, precise kinematic and static positioning, deformation monitoring, and also GNSS-based ionosphere and troposphere study. His research achievements earned him the Heiskanen Senior Award in 2004. In the last several years, he was involved in a number of research projects founded by national and international agencies (e.g., by ESA).

Dr. Pawel Wielgosz is involved in activities of several scientific organizations; he is a member-at-large of the Steering Committee of International Association of Geodesy (IAG) Commission 4 "Positioning \& Applications," vice-chair of IAG Sub-Commission 4.5 "High Precision GNSS Algorithm and Applications." He is an active member of European Geosciences Union (EGU) and American Geophysical Union (AGU). He is also a member of the editorial boards of several scientific journals. 